Chirurg 2006 $\cdot 77: 103-110$ DOI 10.1007/s00104-005-1142-7

Online publiziert: 13. Januar 2006

๑) Springer Medizin Verlag 2006

H.-R. Koelz • M. Arn

Abteilung für Gastroenterologie, Medizinische Klinik, Stadtspital Triemli Zürich

\title{
Neue Epidemiologie der akuten gastrointestinalen Blutung
}

\begin{abstract}
Als Folge der medizinischen Fortschritte würde man erwarten, dass die akuten gastrointestinalen Blutungen in den letzten Jahrzehnten seltener und weniger gefährlich geworden sind. Eine Analyse der epidemiologischen Daten lässt an dieser Hoffnung zweifeln.
\end{abstract}

Viele Neuerungen in der Prophylaxe, Diagnostik und Therapie gastrointestinaler Läsionen der letzten 25 Jahre können als „Meilensteine“ der Gastroenterologie bezeichnet werden: die endoskopische Blutstillung, die Einführung der $\mathrm{H}_{2}$-Blocker und der Protonenpumpeninhibitoren (PPI) und die Wiederentdeckung des H. pylori. Man würde erwarten, dass damit die Häufigkeit und Gefährlichkeit der gastrointestinalen Blutungen reduziert wurde. Andererseits stieg gleichzeitig der Konsum der nichtsteroidale Antirheumatika (NSAR) einschließlich der selektiven COX-2-Hemmer (Coxibe) deutlich an, und das Risiko für Blutungen wurde durch die ständig aggressiver werdenden iatrogenen Gerinnungsstörungen (Antikoagulation, Thrombozytenaggregationshemmung) stark erhöht. Schließlich hatten die Fortschritte der allgemeinen medizinischen Behandlung auch zur Folge, dass die Patienten auch mit schweren Krankheiten länger überleben und damit zunehmend multimorbid werden.

In dieser Übersicht wird der Frage nachgegangen, ob und wie sich diese Veränderungen auf die publizierten epidemiologi- schen Daten aus westlichen Industrienationen ausgewirkt haben. Eine Zusammenstellung der wichtigsten Faktoren, welche die Epidemiologie der gastrointestinalen Blutungen beeinflussen könnten, findet sich in $\bullet$ Tabelle 1.

\section{Einflussfaktoren auf die Epidemiologie}

\section{Ulkusblutung}

Auf die Ulkusblutung wirkt eine Vielzahl von Faktoren ein (• Tabelle 2). Von sehr seltenen Ausnahmen abgesehen, können nur H. pylori und/oder NSAR in Anwesenheit von Magensäure zu Ulzera führen. Bei sorgfältiger Anamnese und Suche nach $\mathrm{H}$. pylori findet sich in $>95 \%$ der Fälle die eine und/oder andere Ursache $[12,23]$.

\section{( In der Regel führen nur H. pylori und NSAR in Anwesenheit von Magensäure zu Ulzera}

In den westlichen Industrieländern nimmt seit Ende des 19. Jahrhunderts die Prävalenz von H. pylori ständig ab [57]. Da der H.-pylori-Infekt in der Kindheit, abhängig vorwiegend von den hygienischen Verhältnissen, stattfindet und spontan nur selten verschwindet, sind heute vorwiegend ältere einheimische Menschen infiziert. Bei jungen Deutschen $[7,25]$ oder Schweizern $[8,19]$ wird ein H.-pylori-Infekt nur noch selten gefunden. Entsprechend nimmt die Helicobacter-bedingte Ulkuskrank- heit in dieser Population ständig ab. Der Rückgang wird durch die seit etwa 1990 weit verbreitete H.-pylori-Eradikationstherapie unterstützt. Dieser Entwicklung entgegen wirken allerdings Immigranten aus Ländern mit noch immer sehr hoher Prävalenz auch bei jungen Personen, beispielsweise aus dem Balkan, der Türkei oder Sri Lanka.

Der andere wichtige Faktor ist der Gebrauch von NSAR, der - vor allem in der älteren weiblichen Bevölkerung - in den letzten 25 Jahren zugenommen hat [27] (- Abb. 1). Da NSAR häufiger zu Magenals zu Duodenalulzera führen, wäre vor allem eine Zunahme der Ulcera ventriculi bei älteren Frauen zu erwarten. Von der Einführung der Coxibe versprach man sich einen Rückgang der NSAR-induzierten Ulkuskrankheit. In vielen Ländern, darunter auch in der Schweiz, in den USA und in Kanada, wurden die nichtselektiven NSAR jedoch nicht durch Coxibe ersetzt, sondern zusätzlich an Patienten verschrieben, die vorher keine NSAR konsumiert hatten. In der Schweiz hatte dies eine Zunahme des NSAR-Konsums um etwa 50\% zur Folge. Da auch die Coxibe das Ulkusrisiko etwas erhöhen und unter Antikoagulation sogar das Blutungsrisiko ähnlich erhöhen wie nichtselektive NSAR [5], erstaunt es nicht, dass in Kanada nach Einführung der Coxibe die Hospitalisationen älterer Patienten wegen Gastrointestinalblutung sogar zunahmen [41]. Nach der Rücknahme von Rofecoxib und Valdecoxib sowie den verschärften Vorschriften für Celecoxib brach der Coxibe-Markt in 


\section{Leitthema}

Tabelle 1

Wichtigste Faktoren, die die Epidemiologie der gastrointestinalen Blutung der letzten 25 Jahre in westlichen Industrieländern beeinflussen können

\section{Faktor}

Zunehmende Überalterung der Bevölkerung

Zunahme des NSAR-Konsums

Verbesserung der Intensivmedizin

Genereller Einsatz der Endoskopie (Gastroskopie, Koloskopie), vor allem auch notfallmäßig

Einführung der $\mathbf{H}_{2}$-Blocker in der Sekundärprophylaxe der Ulkuskrankheit

Prophylaxe der Ösophagusvarizenblutung mit $\beta$-Blockern und endoskopische Sklerotherapie

Weite Verbreitung von Low-dose-Aspirin zur Primärund Sekundärprophylaxe von kardiovaskulären Krankheiten

Einführung der PPI, Anwendung in der Primärund Sekundärprophylaxe der Ulkuskrankheit, v. a. bei NSAR

\section{Eradikationsbehandlung von $\mathbf{H}$. pylori}

Immigration von H.-pylori-positiven Personen aus Risikoländern (z. B. Balkan)

Orale Antikoagulation: Ausweitung der Indikation bei Vorhofflimmern

Vermehrter und kombinierter Einsatz gerinnungshemmender Substanzen (Aspirin, Clopidogrel, Antikoagulation) in der Kardiologie

Varizen: endoskopische Ligaturbehandlung, TIPS

Einführung der Kapselendoskopie

Seit 2000

Ungefährer Zeitraum

Ganze Periode

Ganze Periode

Ganze Periode

Gastroskopie: seit 1975

Koloskopie: seit 1980

1980-1990

Seit 1985

Seit 1985

Seit 1990

Seit 1990

Seit 1990

Seit 1990

Seit 1995

Seit 1995

Einführung der COX-2-selektiven NSAR
Theoretische Auswirkung auf

Blutungsinzidenz

个 Alle Blutungen

个 Mortalität bei Blutung

$\uparrow$ Vorwiegend gastroduodenale Ulzera

个 „Stressulkusblutung“

个 Gesicherte Blutungsursachen

个 Neue Diagnosen wie Angiodysplasien

个 Rezidive (inkl. Blutungen) bei gastroduodenalem Ulkus

个 Erst- und Rezidivblutungen von Ösophagusvarizen

个 Alle Blutungen

$\downarrow$ Gastroduodenale Ulkusblutungen

$\downarrow$ Blutungen aus anderen peptischen Läsionen

$\downarrow$ Gastroduodenale Ulkusblutungen

个 Gastroduodenale Ulkusblutungen

个 Alle Blutungen

个 Alle Blutungen

$\downarrow$ Rezidivblutungen von Ösophagusund (bei TIPS auch) Magenvarizen

个 Identifikation von blutenden Läsionen im Dünndarm

$\downarrow$ Ungesicherte Diagnose "Divertikelblutung"

$\downarrow$ Blutende gastroduodenale Ulzera und Läsionen im unteren Gastrointestinaltrakt

ヤ Zunahme, $\downarrow$ Abnahme. NSAR nichtsteroidale Antirheumatika, PPI Protonenpumpeninhibitoren

Tabelle 2

\section{Einflussfaktoren auf die Epidemiologie des gastroduodenalen Ulkus der letzten 25 Jahre}

\section{Geringer, weil...}

Ulkusinzidenz

$\downarrow$ H.-pylori-Prävalenz bei einheimischer Bevölkerung 个 Gastroprotektion mit PPI oder Misoprostol bei NSAR-Therapie

Blutungsneigung

bestehender Ulzera

Mortalität bei

Ulkusblutung
个 Endoskopische Blutstillung, 个 i.v. PPI-Behandlung

bei akuter Blutung, $\uparrow$ Qualität der medizinischen Betreuung
Höher, weil...

个 H.-pylori-Prävalenz wegen Immigranten aus Risikoländern

$\uparrow$ Konsum von nichtselektiven und COX-2-selektiven NSAR

个 Antikoagulation bei Vorhofflimmern

个 Thrombozytenaggregationshemmer

(Aspirin, Clopidogrel, SSRI)

个 Ältere Patienten

$\uparrow$ Patienten mit schweren Begleiterkrankungen

$\uparrow$ Zunahme bzw. Verbesserung, $\downarrow$ Abnahme, PPI Protonenpumpeninhibitoren, NSAR nichtsteroidale Antirheumatika,

SSRI selektive Serotonin-Reuptake-Inhibitoren. 
der Schweiz zusammen. Die weitere Entwicklung war unerwartet und bedenklich: Fast alle der zuvor mit Coxiben behandelten Patienten wurden auf ein klassisches NSAR umgestellt. Damit steht der Konsum von unselektiven NSAR jetzt auf einem neuen Höchstwert.

\section{Neue Behandlungsmodalitäten bei Ösophagus- und Magenvarizen}

Ebenfalls zu einer Reduktion von Blutungsquellen sollte die Behandlung von gastroösophagealen Varizen führen. Seit etwa 1980 sind wirksame Maßnahmen eingeführt worden, die das Erst- oder Rezidivblutungsrisiko durch Drucksenkung in den Varizen ( $\beta$-Blocker, TIPS) oder lokale Elimination der Varizen (Sklerosierung, Ligatur) vermindern. Diese Methoden sind bei praktisch allen Patienten risikoarm und wirkungsvoll anwendbar. Sie haben die mit einer erheblichen Morbidität und Letalität belasteten chirurgischen Shunts fast vollständig verdrängt.

\section{Zunahme der iatrogenen Blutgerinnungsstörungen}

Blutgerinnungsstörungen erhöhen die Inzidenz und den Schweregrad einer Blutung aus bestehenden Blutungsquellen. Stark zugenommen hat in den letzten beiden Jahrzehnten der prophylaktische Einsatz von oralen (Coumarine) und parenteralen (Heparin, fraktioniertes Heparin) Antikoagulantien sowie von Thrombozytenaggregationshemmern (Low-dose-Aspirin, Clopidogrel usw.).

- Daten aus England, die wohl auch für andere Industrienationen gelten, zeigen innerhalb der Jahre 1990-1999 einen Anstieg der Antikoagulantientherapie auf das Doppelte und der Behandlung mit Low-dose-Aspirin auf fast das Fünffache [27] (• Abb. 1).

Unter Low-dose-Aspirin erhöht sich das gastrointestinale Blutungsrisiko auf ungefähr das Doppelte $[17,52]$, obwohl Aspirin in dieser geringen Dosierung vermutlich nicht zu Ulzera führt [34]. Die zur Behandlung von Depressionen eingesetzten selektiven Serotonin-Reuptake-Inhibitoren (SSRI) sind ebenfalls mit einem erhöh-

Chirurg 2006 $77: 103-110$

DOI 10.1007/s00104-005-1142-7

○) Springer Medizin Verlag 2006

\section{H.-R. Koelz · M. Arn}

\section{Neue Epidemiologie der akuten gastrointestinalen Blutung}

\section{Zusammenfassung}

Die allgemeinen medizinischen, noch mehr aber die gastroenterologischen Fortschritte der letzten 25 Jahre würden erwarten lassen, dass gastrointestinale Blutungen seltener und deren Letalität heute geringer sind. Die publizierten epidemiologischen Daten können dies insgesamt nicht oder nicht im erhofften Ausmaß bestätigen. Die Datenqualität vieler Studien ist aber unbefriedigend. So scheint es beispielsweise, dass fragwürdige Blutungsquellen wie Erosionen im oberen Gastrointestinaltrakt und Kolondivertikel oft wenig kritisch als definitive Ursache der Blutung bezeichnet werden. Klare Hinweise auf Veränderungen finden sich jedoch in Untergruppen. Nach dem Rückgang von $\mathrm{H}$. pylori in der jüngeren Be-

völkerung sind bei der Ulkusblutung heute vermehrt ältere Personen mit zusätzlichen Risikofaktoren wie schwerwiegenden Begleiterkrankungen sowie Einnahme von NSAR, Low-dose-Aspirin und Antikoagulantien betroffen. Nicht unerwartet findet sich in dieser Gruppe generell keine Veränderung oder sogar eine Verschlechterung der Prognose bei akuter Blutung.

\section{Schlüsselwörter}

Epidemiologie · Gastrointestinale Blutung · Helicobacter pylori . Nichtsteroidale Antirheumatika . Protonenpumpeninhibitoren . Ulkuskrankheit · Ösophagusvarizen . Divertikulose

\section{New epidemiology of acute gastrointestinal hemorrhage}

\section{Abstract}

Due to progress in general medicine and especially in gastroenterology, the incidence of acute gastrointestinal hemorrhage could be expected to have decreased during the last 25 years. However, published epidemiological data cannot, in general, fulfill this hope. The interpretation of potential trends is, however, often limited by low study quality. For example, questionable bleeding sources such as erosions in the upper gastrointestinal tract or colon diverticula are often rather uncritically named the definitive causes of bleeding. However, there is clear evidence of changes in grouping of patients. After the almost complete disappearance of Helicobacter pylori in younger indigenous populations of most industrialized countries, it is mostly elderly comorbid people with additional risk factors (NSAID use, low-dose aspirin, anticoagulation) who are affected. Not unexpectedly, this group has generally experienced no change in incidence and in fact shows a deterioration of prognosis in case of acute bleeding.

\section{Keywords}

Epidemiology · Gastrointestinal hemorrhage $\cdot$ Helicobacter pylori . Nonsteroidal anti-inflammatory drugs . Proton pump inhibitors · Gastroduodenal ulcer disease - Gastric ulcer - Duodenal ulcer · Esophageal varices · Diverticulosis 


\section{Leitthema}

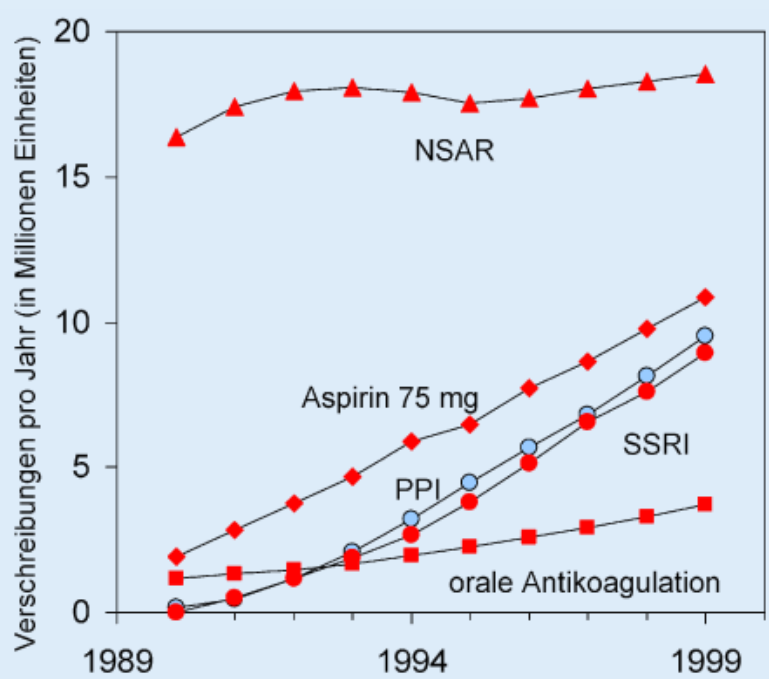

Abb. $1 \Delta$ Verschreibungen von NSAR, Low-dose-Aspirin, oralen Antikoagulantien, PPIs und SSRIs in den Jahren 1990-1999 in England. Daten aus [27]

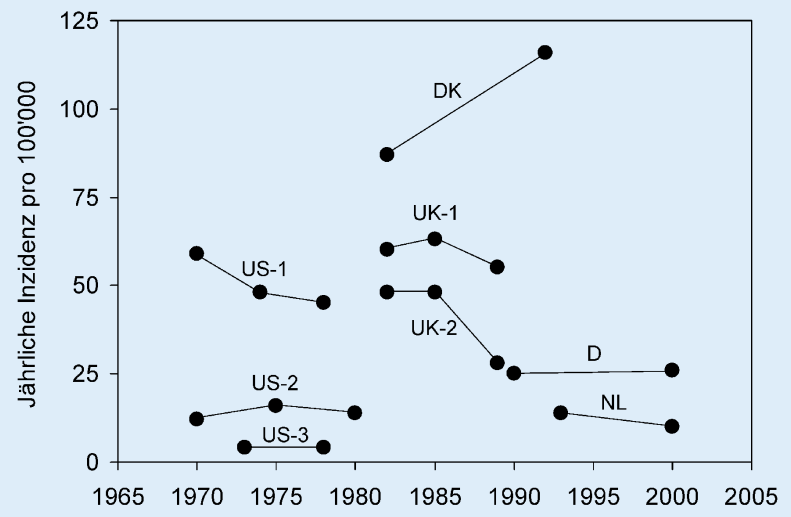

Abb. $3 \Delta$ Inzidenz der Ulkusblutungen. Daten aus Studien, bei denen die populationsbasierte Inzidenz mit gleicher Methodik im Abstand von einigen Jahren wiederholt wurde. Zusammengehörende Werte sind verbunden. Daten aus den USA (US-1 [21], US-2 [33], US-3 [15]), dem United Kingdom (UK-1 und UK-2 [50]), Dänemark (DK [3]), Deutschland (D [47]) und den Niederlanden (NL [62])

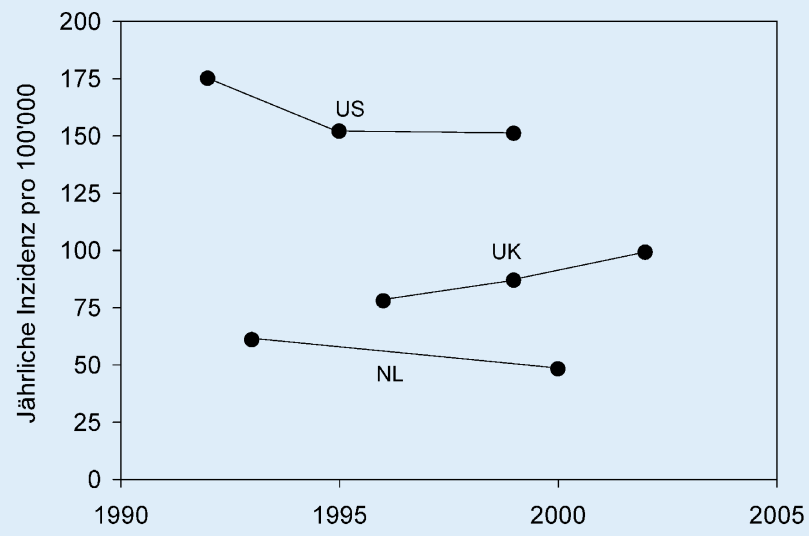

Abb. 2 - Inzidenz der oberen Gastrointestinalblutung. Daten aus Studien, bei denen die populationsbasierte Inzidenz mit gleicher Methodik im Abstand von einigen Jahren wiederholt wurde. Zusammengehörende Werte sind verbunden. Daten aus den USA (US [37]), den Niederlanden (NL [62]) und dem United Kingdom (UK [59])

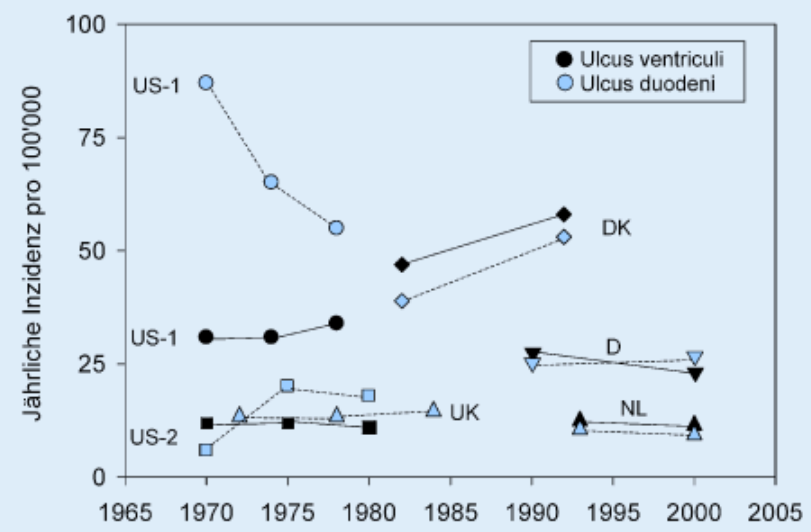

Abb. 4 Inzidenz der Ulkusblutungen, nach Lokalisation unterteilt (ausgefüllte Symbole für Ulcus ventriculi, offene für Ulcus duodeni). Daten aus den US (US-1 [21], US-2 [33]), dem United Kingdom (UK [4]), Dänemark (DK [3]), Deutschland (D [47]) und den Niederlanden (NL [62]) ten Blutungsrisiko assoziiert, vermutlich ebenfalls über eine Hemmung der Thrombozytenaggregation $[27,49]$.

\section{Veränderung von prognostischen Faktoren bei Blutung}

Die zunehmende Überalterung der Bevölkerung und die steigende Multimorbidität verschlechtern die Prognose bei einer Blutung. Eine umgekehrte Wirkung ist aber von den medizinischen Fortschritten in der Behandlung gastrointestinaler Blutungen zu erwarten, beispielsweise der Einführung wirksamer Methoden der endoskopischen Blutstillung (siehe Beitrag von M. Ortner in diesem Heft), der intravenö- sen Therapie mit PPI $[2,35,36]$ sowie der Verbesserung der Intensivmedizin und der Chirurgie.

Neue diagnostische Mittel/ unsichere diagnostische Kriterien

Epidemiologische Daten können durch Änderungen der diagnostischen Kriterien und deren Anwendung stark beeinflusst werden. Daraus resultierende Unterschiede sind natürlich nur scheinbar. $\mathrm{Zu}$ dieser Gruppe gehört die Einführung neuer Methoden wie die der Enteroskopie und der Kapselendoskopie, die sonst nicht erkennbare Blutungsquellen sichtbar machen [32, $42,51,61]$. Noch wichtiger ist wohl die In- terpretation von gewissen Läsionen wie Erosionen des Ösophagus, des Magens und des Duodenums sowie von Kolondivertikeln. Diese können in der Regel nur dann als sichere Blutungsquellen betrachtet werden, wenn eine aktive Blutung direkt beobachtet werden kann.

\section{Epidemiologische Veränderungen der letzten 25 Jahre}

Aus dem Vorhergehenden werden zwei große Probleme der epidemiologischen Daten klar. Erstens sind die zu erwartenden Veränderungen sogar über einen Zeitraum von 25 Jahren vermutlich verhältnismäßig gering, weil die einen Wert be- 
einflussenden Einzelfaktoren häufig in gegensätzliche Richtungen wirken (• Tabelle 1, 2). Zweitens bestehen erhebliche methodologische Schwierigkeiten bei der Datenaufnahme und -interpretation. Sogar wenn das gleiche Patientenkollektiv zweimal unter Anwendung derselben Kriterien analysiert wird, entstehen unvermeidlich scheinbare Unterschiede, die vermutlich größer sind als die zu erwartenden wahren Veränderungen. Am besten sind die wenigen Studien, deren Daten prospektiv, mit demselben Protokoll, an der gleichen Institution und in der gleichen Grundbevölkerung erhoben wurden. Wir stützen uns in der Folge vorwiegend auf solche Publikationen.

\section{Akute obere Gastrointestinalblutung}

Bevölkerungsbasierte Studien über die oberen Gastrointestinalblutungen berichten über eine Inzidenz von etwa 100 pro 100.0oo Einwohner [16, 40, 54, 62], mit einer großen Streubreite von 45 [64] bis $175[6,37]$. Eine Abhängigkeit davon, in welchem Jahr die Daten erhoben wurden, ist nicht erkennbar. Der Grund für die großen Unterschiede liegt vermutlich in den Kriterien zur Definition der akuten Blutung. In der prospektiven Studie mit der geringsten Inzidenz (45 pro 100.000, aus den Niederlanden) fällt mit $63 \%$ eine sehr hohe Häufigkeit von schockierenden Blutungen auf [62]. Die drei Studien $[37,59,62]$, die die Inzidenz im Längsverlauf von 1992-2002 untersuchten, konnten keinen klaren Trend für eine Veränderung finden (• Abb. 2). Vermutlich ebenfalls konstant ist ein eindrücklicher altersabhängiger Unterschied der Inzidenz, indem Personen in der 9. Lebensdekade etwa 30-mal häufiger betroffen sind als solche in der 3. $[26,40]$. Der entscheidende Grund für diese Altersabhängigkeit ist vermutlich die Therapie mit Aspirin und Antikoagulantien [56].

\section{(2) Die Gesamtinzidenz der aku- ten oberen $\mathrm{Gl}$-Blutung hat sich nicht nachweisbar verändert}

Die Letalität der akuten oberen Gastrointestinalblutung jeglichen Ursprungs liegt seit mehreren Jahrzehnten unverändert zwischen 5 und $15 \%[6,54,55,62,64]$.

\section{Verteilung der Blutungsquellen}

Auch die Verteilung der Blutungsquellen zeigt wahrscheinlich keine wesentlichen Veränderungen über die letzten beiden Jahrzehnte. Zwar berichtet eine Studie aus den USA [29] über eine Reduktion des Anteils von gastroduodenalen Ulzera bei oberen Gastrointestinalblutungen von 59\% in den Jahren um 1990 auf 39\% nach 2000. Die in der ersten Untersuchung angegebenen $59 \%$ entsprechen jedoch einem der höchsten je publizierten Werte und sind damit kritisch zu beurteilen; meistens bewegt sich der Anteil der Ulzera um 30$40 \%[6,9,18,40,43,64]$. Eine spezielle Diskussion verdient die publizierte Häufigkeit von Erosionen im Ösophagus, Magen und Duodenum mit einem Bereich von mindestens $7-27 \%$ und - mit diesen Werten oft umgekehrt korrelierend - die Häufigkeit von oberen Gastrointestinalblutungen ohne plausible Blutungsquelle mit 8-24\%. Wenig erfahrene Endoskopiker unter dem Erfolgsdruck, eine Erklärung zu finden, neigen vermutlich öfter zur Diagnose „Blutung aus Erosionen“. Routiniertere und kri- tischere Untersucher bevorzugen hier der Beurteilung „keine klare Blutungsquelle“, zumindest dann, wenn keine gravierende Störung der Blutgerinnung besteht.

Schließlich sei noch das so genannte Stressulkus erwähnt, um das es in den letzten Jahren auffallend ruhig geworden ist. Unter der akuten gastroduodenalen Stressulzeration im engeren Sinn versteht man multiple, vom proximalen Magen sich nach distal bis zum Duodenum ausdehnende Erosionen, die unter extremem physischem Stress (beispielsweise Sepsis, Polytrauma) auftreten und deren gefürchtete Folge eine Blutung war. Typisch für frühere Studien, als die Stressulkusblutung noch als verbreitetes Problem galt, ist, dass die allermeisten Blutungen quantitativ wenig relevant waren und dass die meisten Patienten nicht endoskopiert wurden. Neuere Studien zeigen, dass die meisten relevanten Blutungen nicht aus Erosionen, sondern aus „gewöhnlichen“ Ulzera stattfinden, die - vermutlich vorher schon anwesend - unter den bei Intensivpatienten häufigen Gerinnungsstörungen (spon-

\section{Hier steht eine Anzeige.}

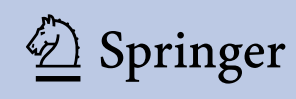



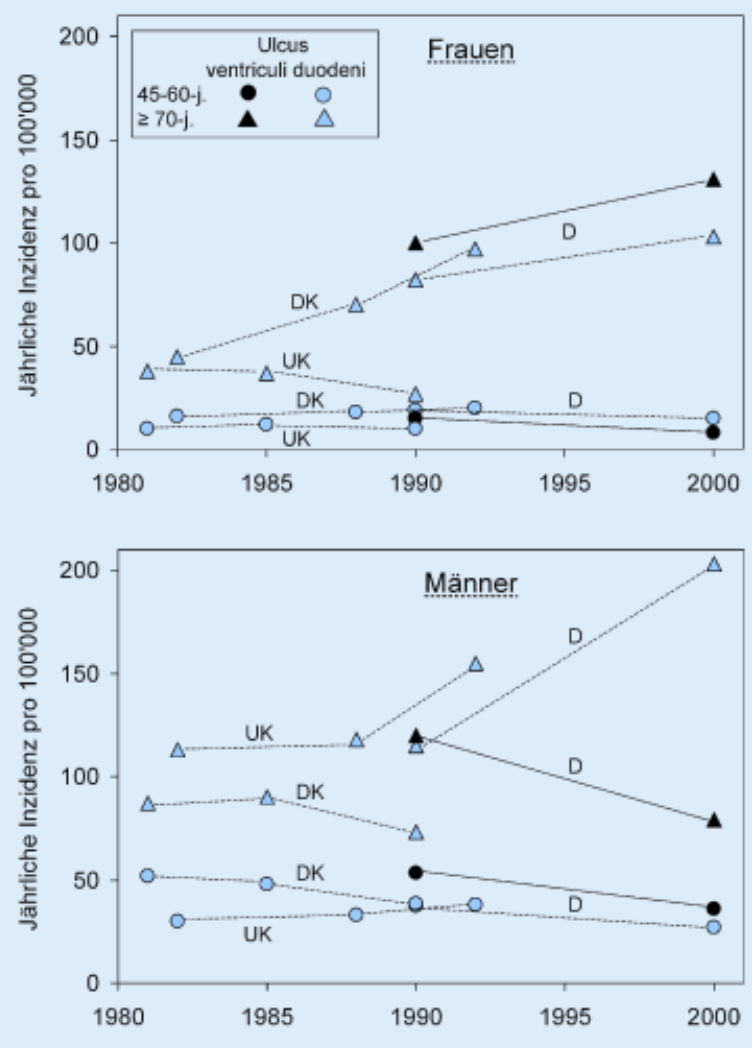

Abb. $5<$ Inzidenz der Ulkusblutungen, nach Geschlecht, Lokalisation und Altersgruppe unterteilt. Daten aus dem United Kingdom [30], Dänemark [3] und Deutschland [47]

tan oder iatrogen) zu bluten beginnen. Es ist damit unklar, ob das fast vollständige Verschwinden der Stressulzeration innerhalb der letzten 25 Jahre auf die verbesserte Intensivmedizin (einschließlich medikamentöse Stressulkusprophylaxe) oder auf eine besser (endoskopische) Diagnostik zurückzuführen ist.

\section{Unkomplizierte Ulzera}

Die unkomplizierte gastroduodenale Ulkuskrankheit ist in den letzten Jahrzehnten insgesamt seltener geworden, vorwiegend als Folge der geringeren Inzidenz bei jüngeren, einheimischen Personen. Da die für die Häufigkeit der Ulkusblutung entscheidenden Risiko- und Schutzfaktoren in verschiedene Richtungen weisen, lässt sich kaum voraussagen, wie die Epidemiologie der Ulkusblutung verläuft.

Es sind 8 Studien verfügbar, in denen die Inzidenz der Ulkusblutungen über mehrere Jahre direkt verglichen wurde (• Abb. 3). Sie zeigen große Differenzen der absoluten Werte für die Inzidenz und unterschiedliche zeitliche Verläufe. Im gleichen Zeitraum, in dem die Inzidenz der Ulkusblutungen in Schottland (UK-1 und UK-2) stark abfällt, zeigt sich ein eindrücklicher Anstieg in Dänemark. Die neuesten Studi- en aus Deutschland und den Niederlanden berichten über einen leichten Rückgang.

Angesichts der vielen Faktoren, welche die Inzidenz der Ulkusblutung in verschiedener Weise beeinflussen könnten ( $\bullet$ Tabelle 1), wäre vorstellbar, dass sich Ulcera ventriculi und Ulcera duodeni unterschiedlich verhalten könnten. Einen derartigen Unterschied weisen nur die Daten einer Studie aus den USA nach (US-1 in - Abb. 4), indem der leichte Rückgang aller Ulkusblutungen der einen Studie aus den USA (US-1) praktisch allein durch eine Abnahme der Ulzera duodeni bedingt war. Ein Rückgang der Inzidenz von Ulzera duodeni um etwa $40 \%$ in der Periode von 1970 bis 1978 ist schwer erklärbar, weil in dieser Zeit keine nennenswerten Änderungen der bekannten Einflussgrößen stattgefunden haben (• Tabelle 1). Die wahrscheinlichste Erklärung für diesen Befund ist eine Änderung der Diagnostik durch die Einführung der Endoskopie, wobei diese Technologie in den USA viel langsamer als in Europa verbreitet wurde. Somit wurden vielleicht in den frühen 7 oer Jahren viele unsichere radiologische $\mathrm{Be}$ funde als Ulcus duodeni interpretiert. Die neueren Daten aus Deutschland und den Niederlanden zeigen immerhin in beiden verfügbaren Studien eine leicht abnehmende oder wenigstens nicht ansteigende Tendenz. Sie erlauben aber kaum eine klare Aussage über allgemeingültige epidemiologische Trends.

\section{(D) Die Risikogruppen haben sich verändert}

Schließlich ist denkbar, dass wichtige Veränderungen in Subgruppen versteckt sind, beispielsweise aufgeteilt nach Geschlecht oder Alter (• Abb. 5). Auch hier sind die Resultate bis 1990 uneinheitlich, und für die Zeit nach 1992 gibt es nur eine einzige Studie [47]. Diese aus Deutschland stammende Arbeit zeigt einerseits den gemäß der H.-pylori-Epidemiologie erwarteten Rückgang aller gastroduodenalen Ulzera bei jüngeren Personen, andererseits einen zum Teil dramatischen Anstieg der Ulkusblutungen bei älteren Menschen, der wenigstens teilweise mit den zunehmenden Verschreibungen von NSAR, Low-doseAspirin und oralen Antikoagulantien in dieser Gruppe korreliert [4, 6, 18, 38, 40, 47, 56, 60, 62, 63]. Der starke Anstieg der Inzidenz von blutenden Ulcera duodeni bei älteren Männern ist jedoch schwer zu erklären. NSAR führen vorwiegend zu Ulcera ventriculi, H. pylori zu Ulcera duodeni. Da ältere Männer weniger NSAR konsumieren als ältere Frauen, sollte sich eigentlich der Erfolg der H.-pylori-Eradikation bei älteren Männern besser manifestieren. Insgesamt ist hier kein einfaches, klares Bild erkennbar.

\section{Veränderungen von Risikofaktoren und der Prognose bei Ulkusblutung. Die Nei-} gung zu Gastrointestinalblutungen hat bei jüngeren Personen abgenommen, bei älteren und mehr noch bei sehr alten Patienten stark zugenommen, zumindest mitbedingt durch den zunehmenden NSARKonsum $[38,46]$ und der immer freizügigeren iatrogenen Hemmung der Blutgerinnung $[45,56]$. Die beiden Einflüsse gleichen sich offenbar für die gesamte Inzidenz etwa aus [47], nicht aber für die Prognose bei Blutung. Die Letalität blieb insgesamt etwa gleich $[13,31,54]$, vor allem bei älteren Patienten, trotz wesentlichen Verbesserungen der Diagnostik und der Therapie. 
Ende der elektiven, aber nicht der notfallmäßigen Ulkuschirurgie. Bereits vor 15 Jahren hat Alexander-Williams das Ende der Vagotomie beziehungsweise der elektiven Ulkuschirurgie prophezeit [1]. Sie ist jetzt tatsächlich praktisch verschwunden $[27,48,50]$. Die Rate der notfallmäßigen Eingriffe für das perforierte [58] oder blutende Ulkus blieb aber konstant oder nahm sogar zu, vor allem bei älteren Frauen [14, 27, 48, 50]. Eine Wirkung der neuen prophylaktischen und therapeutischen Errungenschaften wirkte sich offenbar (noch?) nicht aus - oder kompensiert möglicherweise nur für eine noch ungünstigere Entwicklung.

\section{Ösophagus- und Magenvarizen}

Es gibt keine publizierten prospektiven Studien über den Verlauf der Inzidenz der Varizenblutung über mehrere Jahre. Mehrfach dokumentiert aus Daten des Gesundheitssystems ist jedoch ein Rückgang der Letalität. In einer Studie aus den USA verringerte sich zwischen 1981 und 1991 die Kurzzeitletalität (30 Tage) von 30 auf $21 \%$ und die Langzeitletalität (6 Jahre) von 75 auf $70 \%$, obwohl die spätere Kohorte ein fortgeschritteneres Stadium der Lebererkrankung aufwies [20]. Ähnliche Daten finden sich aus Frankreich zwischen 1987 und 1996 [10] bzw. 1980 und 2000 [11]. Eine Studie zeigt auch eine Verringerung der Mortalität an Varizenblutungen in der Gesamtbevölkerung [10].

\section{Akute untere \\ Gastrointestinalblutung}

Über die untere gastrointestinale Blutung gibt es viel weniger Daten als über die obere. Insbesondere fehlen epidemiologische Längsverläufe guter Qualität.

Üblicherweise unterscheidet man bei Blutungen zwischen einem oberen und einem unteren Gastrointestinaltrakt, wobei die Grenze beim Treitz-Ligament liegt. Im unteren Gastrointestinaltrakt war - bei kritischer Beurteilung - ein größerer Anteil von Blutungsquellen nicht oder nicht sicher identifizierbar. Dies lag möglicherweise daran, dass fast der gesamte Dünndarm bisher endoskopisch nicht zugänglich war. Mit der Verbreitung der Push- und Doppelballonenteroskopie sowie der Kapselendoskopie hat sich die Situation geändert, und es wurde bereits vorgeschlagen, vom unteren Gastrointestinaltrakt einen „mittleren “ abzugrenzen, der den Dünndarm vom Jejunum bis zum Ileum umfasst [51].

\section{Inzidenz}

Die publizierte populationsbasierte jährliche Inzidenz relevanter akuter unterer Gastrointestinalblutungen schwankt zwischen 20 und 30 pro 100.000 Einwohner und ist damit 4- bis 6-mal seltener als die Blutung aus dem oberen Gastrointestinaltrakt [22]. Noch deutlicher als bei der oberen Blutung zeigt sich hier eine Altersabhängigkeit: Das mittlere Alter dieser Patienten beträgt etwa 70 Jahre; im 9. Lebensjahrzehnt ist sie etwa 200-mal häufiger als im 3. [22, 39].

\section{Verteilung der Blutungsquellen}

Die in den allermeisten Studien am häufigsten gefundene Blutungsquelle ist die Kolondivertikulose, die für $20-50 \%$ der Fälle verantwortlich gemacht wird [22], im Allgemeinen gefolgt von Angiodysplasien in 3-40\%. Der extrem weite Bereich dieser Angaben ist auf die große Unsicherheit der Diagnosen zurückzuführen. Dieser Ansicht entsprechen auch die fast unglaublichen Schwankungen der Angaben (12-41\%) zu den Fällen, in denen keine Blutungsquelle gefunden werden konnte [22, $24,39,44,53]$. Die sichere Identifikation einer Läsion als Blutungsquelle, das heißt die direkte Beobachtung einer blutenden Läsion, ist - vorwiegend aus technischen Gründen - im unteren Gastrointestinaltrakt sehr viel schwieriger und seltener als im oberen Gastrointestinaltrakt.

\section{(D Gesicherte Divertikelblutungen sind selten}

So wird aus der bloßen Anwesenheit von Divertikeln, wie sie bei älteren Menschen auch ohne Blutung in über 50\% vorkommen, eher leichtfertig die Diagnose Divertikelblutung gestellt. Da die unteren - wie die oberen - gastrointestinalen Blutungen in etwa $80 \%$ spontan und definitiv sistieren, wird man selten zu einer Korrektur der Diagnose gezwungen. Eine zusätzliche Schwierigkeit besteht darin, dass lokale Zeichen, die auf eine kürzliche Blutung hinweisen, bei Divertikeln schlecht definiert und validiert sind. Eine neuere
Studie, bei der Patienten mit unterer Gastrointestinalblutung innerhalb von höchstens $8 \mathrm{~h}$ nach Krankenhauseintritt koloskopiert wurden, verdeutlicht dieses Problem [24]: Bei nicht weniger als 39 von 50 Patienten (79\%) wurde als Blutungsquelle eine Kolondivertikulose angeschuldigt. Die Diagnose wurde bei 26 (52\%) als ,vermutlich“ und in 13 Fällen (17\%) als „sicher“ angegeben. $\mathrm{Zu}$ der Gruppe mit einer „sicheren“ Blutungsquelle gehörten 8 mit aktiver Blutung und 5 mit anderen Blutungsstigmata. Eine andere, ähnliche Studie fand bei 121 im Mittel 66-jährigen Patienten mit Kolondivertikulose nur bei $12 \%$ eine ,vermutliche“ und bei $22 \%$ eine "definitive“ Divertikelblutung, insgesamt nur in $9 \%$ mit aktiver Blutung [28]. Es scheint somit, dass allein eine kritischere Diagnostik die Kolondivertikulose vom ersten Platz der Blutungsquellen verdrängt. Unter den identifizierten Läsionen wird dieser heute vermutlich von Angiodysplasien eingenommen [28].

Mit der Einführung der Enteroskopie und der Kapselendoskopie, wird sich das Spektrum der (diagnostizierten) Blutungsquellen im Dünndarm bestimmt ändern [42]. Bevor diese Methoden breiter angewandt wurden, galt der Dünndarm des Erwachsenen als ein Bereich mit einer geringen Wahrscheinlichkeit für den Ursprung einer Blutung. Dies wurde vorwiegend aus der bescheidenen diagnostischen Ausbeute der Kontrastmitteldarstellungen nach Sellink und der Angiographie geschlossen. Vor allem die Kapselendoskopie hat eine erstaunlich Häufigkeit von Läsionen im Dünndarm zeigen können, allerdings auch bei völlig asymptomatischen jungen Gesunden.

Es ist mit guten Gründen anzunehmen, dass einige Fälle mit der früheren Beurteilung „untere Gastrointestinalblutung mit unklarer Blutungsquelle" durch eindeutige Läsionen im Dünndarm erklärt werden - und hoffentlich auch einige derjenigen Patienten mit der Vermutungsdiagnose „Divertikelblutung“ oder Angiodysplasie.

\section{Fazit für die Praxis}

\section{Die epidemiologischen Daten über die akute gastrointestinale Blutung der letz- ten 25 Jahre sind verhältnismäßig spär- lich, vielfach von zweifelhafter Qualität}


und oft kontradiktorisch. Sie sind deshalb schwer interpretierbar. Trotzdem sind einige Tendenzen offensichtlich: Die bevölkerungsbasierte Inzidenz und die Prognose bei Blutung haben sich - mit Ausnahme der Letalität bei Varizenblutung - nicht oder nicht wesentlich verändert. Es hat jedoch eine Verschiebung der Betroffenen stattgefunden: Patienten mit Blutungen sind heute älter, weisen eine höhere Komorbidität auf, konsumieren öfter NSAR und werden häufiger ( $a b-$ sichtlich) mit Substanzen behandelt, die die Blutgerinnung stören. Es scheint also, dass die zweifellos großen Fortschritte der gastroenterologischen Prophylaxe und der Therapie durch zusätzliche Risiken „,maskiert“ werden. Das Blutungsrisiko könnte durch eine strenge Indikation der Verschreibung von NSAR (einschließlich Coxiben), Thrombozytenaggregationshemmer und Antikoagulantien vor allem bei der älteren Bevölkerung vermindert werden.

\section{Korrespondierender Autor Prof. Dr. H.-R. Koelz}

Abteilung für Gastroenterologie, Medizinische Klinik, Stadtspital Triemli, Birmensdorferstrasse 497, CH-8063 Zürich E-Mail: hansrudolf.koelz@triemli.stzh.ch

Interessenkonflikt: Der korrespondierende Autor ist Consultant bei AstraZeneca Schweiz. Andere Verbindungen mit einem hier genannten Produkt oder einem Konkurrenzprodukt bestehen nicht. Die Präsentation des Themas ist unabhängig und die Darstellung der Inhalte produktneutral.

\section{Literatur}

1. Alexander-Williams J (1991) A requiem for vagotomy. Despite the last ditch efforts of surgeons (editorial). BMJ 302:547-548

3. Bak Andersen I, Bonnevie O, Jörgensen T, Sörensen TIA (1998) Time trends for peptic ulcer disease in Denmark, 1981-1993. Scand J Gastroenterol 33:260-266

4. Bardhan KD, Cust G, Hinchliffe RF, Williamson FM, Lyon C, Bose K (1989) Changing pattern of admissions and operations for duodenal ulcer. Br J Surg 76:230-236

5. Battistella M, Mamdami MM, Juurlink DN, Rabeneck L, Laupacis A (2005) Risk of upper gastrointestinal hemorrhage in warfarin users treated with nonselective NSAIDs or COX-2 inhibitors. Arch Intern Med 165:189-192

11. Carbonell N, Pauwels A, Serfaty L, Fourdan O, Levy VG, Poupon R (2004) Improved survival after variceal bleeding in patients with cirrhosis over the past two decades. Hepatology 40:652-659
17. Derry S, Loke YK (2000) Risk of gastrointestinal haemorrhage with long term use of aspirin: meta-analysis. BMJ 321:1183-1187

20. El-Serag HB, Everhart JE (2000) Improved survival after variceal hemorrhage over an 11-year period in the Department of Veterans Affairs. Am J Gastroenterol 95:3566-3573

21. Elashoff JD, Grossman MI (1980) Trends in hospital admissions and death rates for peptic ulcer in the United States from 1970 to 1978. Gastroenterology 78:280-285

22. Farrell JJ, Friedman LS (2005) Review article: the management of lower gastrointestinal bleeding. Aliment Pharmacol Ther 21:1281-1298

24. Green BT, Rockey DC, Portwood G et al. (2005) Urgent colonoscopy for evaluation and management of acute lower gastrointestinal heamorrhage: $A$ randomized controlled trial. Am J Gastroenterol 100:2395-2402

27. Higham J, Kang JYMA (2002) Recent trends in admission and mortality due to peptic ulcer in England: Increasing frequency of haemorrhage among older subjects. Gut 50:460-464

28. Jensen DM, Machicado $G A$, Jutabha R, Kovacs $T O$ (2000) Urgent colonoscopy for the diagnosis and treatment of severe diverticular hemorrhage. $\mathrm{N}$ Engl J Med 342:78-82

29. Jensen DM, Mawas I, Lousuebsakul V, Fontana L, St Amand A (2003) Changes in the prevalence of different diagnosies for UGI hemorrhage in the last two decades (abstract). Gastrointest Endosc 57:AB147

30. Jibril JA, Redpath A, Macintyre IM (1994) Changing pattern of admission and operation for duodenal ulcer in Scotland. Br J Surg 81:87-89

33. Kurata JH, Honda GD, Frankl H (1982) Hospitalization and mortality rates for peptic ulcers: a comparison of a large health maintenance organization and United States data. Gastroenterology 83:10081016

34. Laine L, Maller ES, Yu C, Quan H, Simon T (2004) UIcer formation with low-dose enteric-coated aspirin and the effect of COX-2 selective inhibition: a double-blind trial. Gastroenterology 127:395-402

37. Lewis JD, Bilker WB, Brensinger C, Farrar JT, Strom BL (2002) Hospitalization and mortality rates from peptic ulcer disease and GI bleeding in the $1990 \mathrm{~s}$ : relationship to sales of nonsteroidal anti-inflammatory drugs and acid suppression medications. Am J Gastroenterol 97:2540-2549

38. Lim CH, Heatley RV (2005) Prospective study of acute gastrointestinal bleeding attributable to anti-inflammatory drug ingestion in the Yorkshire region of the United Kingdom. Postgrad Med J 81:252254

41. Mamdani M, Juurlink DN, Kopp A, Naglie G, Austin PC, Laupacis A (2004) Gastrointestinal bleeding after the introduction of COX-2 inhibitors: ecological study. BMJ 328:1415-1416

46. Ofman JJ, MacLean CH, Straus WL, Morton SC, Berger ML, Roth EA, Shekelle P (2002) A metaanalysis of severe upper gastrointestinal complications of nonsteroidal antiinflammatory drugs. J Rheumatol 29:804-812

47. Ohmann C, Imhof M, Ruppert C et al. (2005) Timetrends in the epidemiology of peptic ulcer bleeding. Scand J Gastroenterol 40:914-920

48. Paimela H, Oksala NK, Kivilaakso E (2004) Surgery for peptic ulcer today. A study on the incidence, methods and mortality in surgery for peptic ulcer in Finland between 1987 and 1999. Dig Surg 21:185-191
50. Penston JG, Crombie IK, Waugh NR, Wormsley KG (1993) Trends in morbidity and mortality from peptic ulcer disease: Tayside versus Scotland. Aliment Pharmacol Ther 7:429-442

51. Prakash C, Zuckerman GR (2003) Acute small bowel bleeding: a distinct entity with significantly different economic implications compared with Gl bleeding from other locations. Gastrointest Endosc 58:330-335

52. Ridker PM, Cook NR, Lee IM et al. (2005) A randomized trial of low-dose aspirin in the primary prevention of cardiovascular disease in women. $\mathrm{N}$ Engl J Med 352:1293-1304

56. Sam C, Massaro JM, D'Agostino RB Sr, Levy D, Lambert JW, Wolf PA, Benjamin EJ (2004) Warfarin and aspirin use and the predictors of major bleeding complications in atrial fibrillation (the Framingham Heart Study). Am J Cardiol 94:947-951

57. Sonnenberg A (1995) Temporal trends and geographical variations of peptic ulcer disease. Aliment Pharmacol Ther 9 Suppl 2:3-12

59. Taha AS, Angerson WJ, Naur S, Knill-Jones RP, Blatchford O (2005) Upper gastrointestinal haemorrhage associated with low-dose aspirin and anti-thrombotic drugs - a six-year epidemiological analysis and comparison with non-steroidal anti-inflammatory drugs. Gastroenterology 128 [Suppl 2]: A-154

62. Van Leerdam ME, Vreeburg EM, Rauws EA, Geraedts AA, Tijssen JG, Reitsma JB, Tytgat GN (2003) Acute upper GI bleeding: did anything change? Time trend analysis of incidence and outcome of acute upper Gl bleeding between 1993/1994 and 2000. Am J Gastroenterol 98:1494-1499

64. Vreeburg EM, Snel P, de Bruijne JW, Bartelsman JFWM, Rauws EAJ, Tytgat GNJ (1997) Acute upper gastrointestinal bleeding in the Amsterdam area: Incidence, diagnosis, and clinical outcome. Am J Gastroenterol 92:236-243

Das komplette Literaturverzeichnis ..

... finden Sie in der elektronischen Version dieses Beitrags unter DerChirurg.de 